\title{
One-Dimensional Pricing of CPPI
}

\author{
Louis Paulot and Xavier Lacroze
}

\author{
Sophis Technology \\ 24-26 place de la Madeleine, 75008 Paris, France \\ louis.paulot@sophis.net \\ xavier.lacroze@sophis.net
}

May 18, 2009

\begin{abstract}
Constant Proportion Portfolio Insurance (CPPI) is an investment strategy designed to give participation in the performance of a risky asset while protecting the invested capital. This protection is however not perfect and the gap risk must be quantified. CPPI strategies are path-dependent and may have American exercise which makes their valuation complex. A naive description of the state of the portfolio would involve three or even four variables. In this paper we prove that the system can be described as a discrete-time Markov process in one single variable if the underlying asset follows a homogeneous process. This yields an efficient pricing scheme using transition probabilities. Our framework is flexible enough to handle most features of traded CPPIs including profit lock-in and other kinds of strategies with discrete-time reallocation.
\end{abstract}

Keywords: CPPI, Portfolio Insurance, Option, Pricing, Gap Risk, Markov. 


\section{Contents}

1 Introduction $\quad 2$

2 Basic algorithm 4

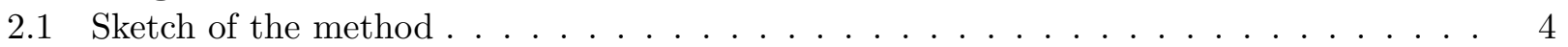

2.2 Mathematical formulation . . . . . . . . . . . . . . . . 6

2.3 Numerical implementation . . . . . . . . . . . . . . . . . . . 7

2.4 Numerical convergence . . . . . . . . . . . . . . . . . . . 7

3 Profit lock-in 8

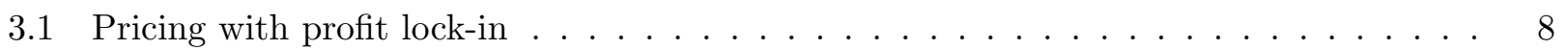

3.2 Numerical implementation . . . . . . . . . . . . . . . . . . . . 10

3.3 Probability transition .......................... 11

4 Numerical examples 12

5 Conclusion $\quad \mathbf{1 2}$

\section{Introduction}

Constant Proportion Portfolio Insurance is a dynamic strategy designed to give participation in risky assets while protecting the invested capital [Mer71. This is achieved by periodically rebalancing between a risk-free asset (Zero-Coupon bond) and a risky asset (share, index, fund, fund of funds...). In the simplest form, if the underlying asset has no jumps and if one can rebalance continuously, the final payoff depends in a deterministic way on the risky underlying. However both hypotheses are strong and do not fit real market conditions. If they are relaxed, the strategy is not as efficient: there is a small chance of not recovering at maturity the invested capital. This gap risk may be held by the issuer, so that the principal is really guaranteed to the investor. In this case, there is an option included in the product, which must be priced and hedged. For a very simple CPPI strategy with continuous rebalancing, the gap risk comes only from instantaneous jumps and can be quantified analytically [CT07]. With a discrete rebalancing scheme, there is a closed formula for the embedded option price if the underlying follows a Black-Scholes diffusion [BBM05]. This formula can as well be generalized to jump-diffusion models, and more generally to Levy processes [PL09]. However these methods work only for an idealized CPPI product where there are no caps on the risky exposure, no spreads on the risk-free and financing rates, no fees, no profit lock-in, a natural bond floor... Unfortunately, real CPPIs have usually such features, which prevent from using closed formulas. Moreover, such formulas does not hold for options with strikes differing from the guaranteed amount. This makes necessary to use other methods for real-life, more complex products. As CPPIs are very path dependent, they are usually priced through Monte-Carlo simulations (see [BK95] for an example). Extreme value theory has also been used to estimate the gap risk of such products [BP02].

Monte-Carlo pricing is perfectly suited to path dependence but the dimension of the problem is generally quite large. As an example, a monthly CPPI defined on a single risky asset with monthly rebalancing and a 20 years maturity requires simulating 240 values of the underlying. This means a 240-dimensional Monte-Carlo integration. Furthermore, the tails of the distributions are crucial in the pricing. First of all, the lower tail gives the gap risk part. It must be computed with enough precision to produce a reasonable Put price and an accurate Delta. The upper tail is important to reach the correct mean value of the CPPI strategy. The payoff distribution is close to a shifted lognormal distribution with high leverage: most trajectories will end below the mean and will be compensated 
by a few very high terminal values. The high path dependence and barrier-like structure of the strategy makes the Put price and its Delta converge very slowly. As an illustration, we refer to figure 1 in which we present an example of the convergence of a put option on the CPPI portfolio: after 20 millions trajectories (and hours of computation), the Monte-Carlo method obviously do not yield a satisfactory estimation of the price of the derivative.

An other way to price CPPI derivatives would be to use a PIDE scheme. This would also handle the American or more precisely Bermudan exercise which is more and more present in such products. However, there are at least three variables to propagate which depend on each other: the risky underlying value, the CPPI portfolio value and the risk asset weighting. If some profit lock-in feature is present, the guarantee level increases the dimension of the PIDE to four. The high dimensionality combined with the large number of time steps needed (at least the number of rebalancings) makes this solution very computationally expensive.

There are drastic simplifications if one only considers the portfolio value only at rebalancing times. We prove that if the underlying asset follows an homogeneous process, the dynamics of the portfolio at rebalancing dates is described by a discrete time Markov process in one single variable. This property leads to a simple and efficient numerical scheme. The main advantages of this technique are its speed, the smoothness of greeks and the ability to handle Bermudan exercise. For example in the simplest cases, only a few milliseconds are needed to get an accurate price. More complex cases with profit lock-in, artificial cushion or coupons take more time but never more than a few seconds.

In section 2, we start by describing our pricing method in simple cases for which the guarantee amount is constant: after introducing our working hypothesis, we formulate the main result concerning

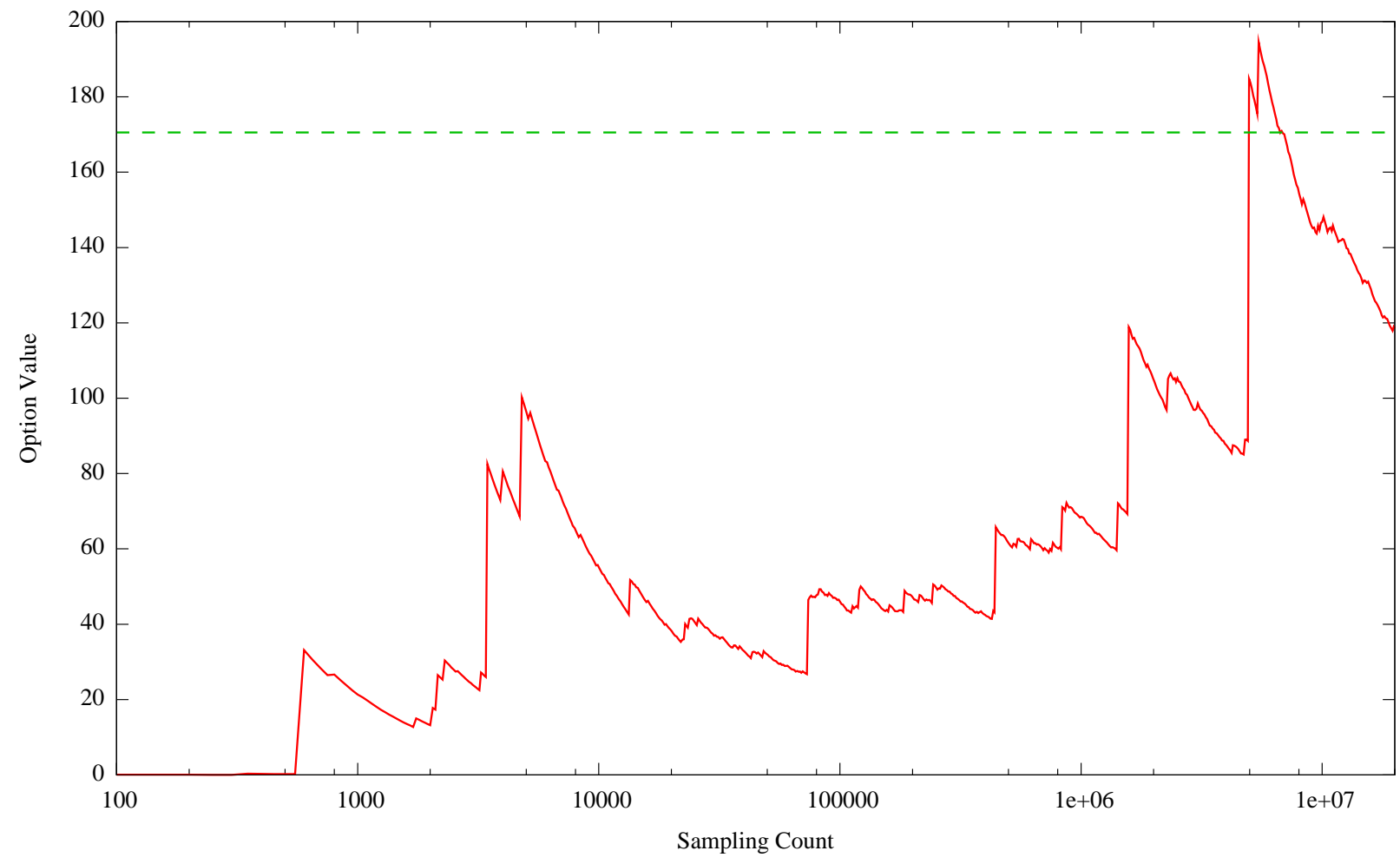

Figure 1: Convergence of the Monte Carlo method for the price of a put on a vanilla CPPI portfolio as functions of the number of paths. Maturity is 10 years, multiplier is 4, rebalancing is performed weekly, volatility is 50\%, risk-free rate is 5\% and initial investment is 1000000 . The strike is set to this value. The dashed line indicates the exact value obtained by analytical computation. 
the transition probabilities of the CPPI portfolio at rebalancing dates and finally discuss the numerical implementation of this method. In section 3, we allow the guaranteed amount to vary over time through a feature called "profit lock-in". Under some homogeneity assumption on the rebalancing rule, we show that the problem is still one-dimensional. Again, we briefly discuss the steps involved in the numerical implementation.

\section{Basic algorithm}

\subsection{Sketch of the method}

At a time $t$, we consider a portfolio (the CPPI portfolio) with total value $C(t)$. This portfolio is split between a risky and a risk-free components:

$$
C(t)=C^{\text {risky }}(t)+C^{\text {risk-free }}(t)
$$

The risk-free part is invested in zero-coupons of same maturity as the CPPI, $B(t)$, whose dynamic is completely deterministic

$$
B\left(t^{\prime}\right)=B(t) e^{r\left(t^{\prime}-t\right)}
$$

with $r$ the risk-free interest rate (taken constant for simplicity). The risky part is invested in an asset $S(t)$. For simplicity in this part we consider a Black-Scholes dynamics which under the risk-neutral measure is

$$
\mathrm{d} S(t)=r S(t) \mathrm{d} t+\sigma S(t) \mathrm{d} W(t)
$$

A CPPI strategy is designed to protect some part of the investment and we shall denote by $G$ this "guaranteed" amount. At this point, $G$ is just some constant, which is generally taken to be the initial value of the portfolio $C(0)$. Taking a fraction $1 / G$ of the portfolio, we can consider without loss of generality a unit guarantee. In this section we take therefore $G=1$.

Let $t_{0}=0<t_{1}<\cdots<t_{n}$ be some fixed times, called rebalancing dates. At each rebalancing date $t_{i}$, the allotment of the portfolio between the risky and risk-free components is adjusted (without any change in the total value of the portfolio) according to a rule specified through a non-negative deterministic function $w(t, x)$ called the risky asset weighting (or simply RAW):

$$
C^{\text {risky }}\left(t_{i}\right)=w\left(t_{i}, C\left(t_{i}\right)\right) C\left(t_{i}\right)
$$

For a CPPI strategy, $w$ is usually chosen of the form

$$
w(t, C)=\max \left(m \frac{C-H(t)}{C}, 0\right)
$$

where $m$ is called the multiplier of the strategy and $H$ is a positive deterministic function called the floor whose terminal value is the guarantee (equal to 1 here). A natural choice is to set the floor to be

$$
H(t)=e^{-r\left(t_{n}-t\right)}
$$

$H(t)$ is equal to the value of a (unit nominal) zero-coupon bond one must hold to recover 1 at maturity $t_{n}$. Within this setup, this investment strategy indeed offers some protection: when the portfolio poorly performs the investment is smoothly allocated to the risk-free asset. With continuous rebalancing and without jumps in the asset price, the bond floor cannot be breached. With discrete rebalancing there is still a probability of going under the floor: the floor is breached if the risky asset goes down by more than $1 / m$ between two rebalancing dates. 
Between rebalancing dates, the composition of the portfolio is left unchanged. For $t \in\left[t_{i}, t_{i+1}\right]$, this reads

$$
C(t)=C^{\text {risky }}\left(t_{i}\right) \frac{S(t)}{S\left(t_{i}\right)}+C^{\text {risk-free }}\left(t_{i}\right) \frac{B(t)}{B\left(t_{i}\right)}
$$

In the case $t=t_{i+1}$, this equation gives the evolution of the portfolio between two consecutive rebalancing dates:

$$
C\left(t_{i+1}\right)=C^{\text {risky }}\left(t_{i}\right) \frac{S\left(t_{i+1}\right)}{S\left(t_{i}\right)}+C^{\text {risk-free }}\left(t_{i}\right) \frac{B\left(t_{i+1}\right)}{B\left(t_{i}\right)}
$$

or equivalently, eliminating $C^{\text {risky }}$ using equation (1),

$$
C\left(t_{i+1}\right)=w\left(t_{i}, C\left(t_{i}\right)\right) C\left(t_{i}\right) \frac{S\left(t_{i+1}\right)}{S\left(t_{i}\right)}+\left(1-w\left(t_{i}, C\left(t_{i}\right)\right)\right) C\left(t_{i}\right) \frac{B\left(t_{i+1}\right)}{B\left(t_{i}\right)}
$$

Then, at date $t_{i+1}$, the manager computes the new Risky Asset Weighting $w\left(t_{i+1}, C\left(t_{i+1}\right)\right)$ and reallocates the resources. Once again, we emphasize that this rebalancing procedure has no instantaneous effect on the total value of the portfolio.

In a first step, we look at the risk-neutral probability distribution of the portfolio value over a single rebalancing period. It can be seen from equation (4) that $C\left(t_{i+1}\right)$ depends on $C\left(t_{i}\right)$ and on the ratio of the risky asset values $S\left(t_{i+1}\right) / S\left(t_{i}\right)$. However, if the process $S(t)$ is homogeneous (which clearly is the case of the lognormal dynamic we consider here), this ratio does not depend on $S\left(t_{i}\right)$ itself. If in addition we suppose that the risky asset has independent increments, the distribution of $C\left(t_{i+1}\right)$ conditionally on time $t_{i}$ depends only on $C\left(t_{i}\right)$ :

$$
\begin{aligned}
\mathbb{P}\left[C\left(t_{i+1}\right)<y \mid \mathcal{F}_{t_{i}}\right] & =\mathbb{P}\left[C\left(t_{i+1}\right)<y \mid C\left(t_{i}\right)\right] \\
& =\mathbb{P}\left[\frac{S\left(t_{i+1}\right)}{S\left(t_{i}\right)}<\frac{y-\left(1-w\left(t_{i}, C\left(t_{i}\right)\right)\right) C\left(t_{i}\right) B\left(t_{i+1}\right) / B\left(t_{i}\right)}{w\left(t_{i}, C\left(t_{i}\right)\right) C\left(t_{i}\right)} \mid C\left(t_{i}\right)\right]
\end{aligned}
$$

This equation relates the distribution of $C\left(t_{i+1}\right)$ conditionally on $C\left(t_{i}\right)$ to the distribution of $S\left(t_{i+1}\right) / S\left(t_{i}\right)$. In the case of Black-Scholes diffusion that we consider, the cumulative function is

$$
\mathbb{P}\left[\frac{S\left(t_{i+1}\right)}{S\left(t_{i}\right)}<z\right]=\mathcal{N}\left(\frac{\ln z-\left(r-\frac{1}{2} \sigma^{2}\right)\left(t_{i+1}-t_{i}\right)}{\sigma \sqrt{t_{i+1}-t_{i}}}\right)
$$

Combining these two equations we get an explicit formula for $\mathbb{P}\left[C\left(t_{i+1}\right)<y \mid \mathcal{F}_{t_{i}}\right]$ as a function of $C\left(t_{i}\right)$. The process for the portfolio value at rebalancing times is therefore a Markov process in discrete time. The general framework for the study of Markov processes leads to introducing the risk-neutral probability density of $C\left(t_{j}\right)$ conditionally on $C\left(t_{i}\right)$, also called transition kernel for the period $\left[t_{i}, t_{i+1}\right]$ :

$$
\phi_{t_{i}, t_{i+1}}(x, y)=\frac{\partial}{\partial y} \mathbb{P}\left[C\left(t_{i+1}\right)<y \mid C\left(t_{i}\right)=x\right]
$$

which is computed explicitly using equations (5) and (5).

Let us consider a European derivative product $V$ written on the CPPI portfolio with terminal payoff at $t_{n}$ given by

$$
V\left(t_{n}, C\left(t_{n}\right)\right)=P\left(C\left(t_{n}\right)\right)
$$

If we know the value of this derivative at time $t_{i+1}$ for all values of $C\left(t_{i+1}\right)$, its value at time $t_{i}$ and CPPI value $C\left(t_{i}\right)$ is given by the discounted risk-neutral expected value

$$
\begin{aligned}
V\left(t_{i}, C\left(t_{i}\right)\right) & =e^{-r\left(t_{i+1}-t_{i}\right)} \mathbb{E}\left[V\left(t_{i+1}, C\left(t_{i+1}\right)\right) \mid C\left(t_{i}\right)\right] \\
& =e^{-r\left(t_{i+1}-t_{i}\right)} \int \mathrm{d} y \phi_{t_{i}, t_{i+1}}\left(C\left(t_{i}\right), y\right) V\left(t_{i+1}, y\right)
\end{aligned}
$$


Starting from the terminal payoff and applying recursively this formula yields today fair price of the derivative $V\left(t_{0}, C\left(t_{0}\right)\right)$.

This backward recursion is also suited to Bermudan exercise (at rebalancing dates):

$$
V\left(t_{i}, C\left(t_{i}\right)\right)=\max \left(e^{-r\left(t_{i+1}-t_{i}\right)} \int \mathrm{d} y \phi_{t_{i}, t_{i+1}}\left(C\left(t_{i}\right), y\right) V\left(t_{i+1}, y\right), P\left(C\left(t_{i}\right)\right)\right)
$$

\subsection{Mathematical formulation}

We collect the previous results (with slightly more general hypothesis) in the following proposition.

Proposition 1. Let us consider a CPPI portfolio with a given set of rebalancing dates $t_{0}, \ldots, t_{n}$ and guaranteed amount $G$. If the following hypotheses hold:

- the risky asset weighting is given by a deterministic function $w(t, C / G)$;

- the underlying risky asset follows a one-dimensional homogeneous process with cumulative distribution function

$$
\mathcal{Q}_{t, t^{\prime}}(z)=\mathbb{P}\left[\frac{S\left(t^{\prime}\right)}{S(t)}<z\right]
$$

which depends only on $t, t^{\prime}$ and $z$;

- the risk free asset is deterministic with value $B(t)$;

then the CPPI portfolio value taken at rebalancing dates $C\left(t_{i}\right)$ is a Markov process in discrete time. The probability transition operator of the normalized variable $C\left(t_{i}\right) / G$

$$
\phi_{t_{i}, t_{i+1}}(x, y)=\mathbb{E}\left[\delta\left(\frac{C\left(t_{i+1}\right)}{G}-y\right) \mid \frac{C\left(t_{i}\right)}{G}=x\right]
$$

given by

$$
\phi_{t_{i}, t_{i+1}}(x, y)= \begin{cases}\frac{\partial}{\partial y} \mathcal{Q}_{t_{i}, t_{i+1}}\left[\frac{y-\left(1-w\left(t_{i}, x\right)\right) x B\left(t_{i+1}\right) / B\left(t_{i}\right)}{w\left(t_{i}, x\right) x}\right] & \text { if } w\left(t_{i}, x\right)>0 \\ \delta\left(y-x \frac{B\left(t_{i+1}\right)}{B\left(t_{i}\right)}\right) & \text { if } w\left(t_{i}, x\right)=0\end{cases}
$$

European derivatives on the CPPI portfolio are valued recursively through

$$
V\left(t_{i}, C\left(t_{i}\right)\right)=e^{-r\left(t_{i+1}-t_{i}\right)} \int \mathrm{d} y \phi_{t_{i}, t_{i+1}}\left(\frac{C\left(t_{i}\right)}{G}, y\right) V\left(t_{i+1}, G y\right)
$$

and Bermudan derivatives using

$$
V\left(t_{i}, C\left(t_{i}\right)\right)=\max \left(e^{-r\left(t_{i+1}-t_{i}\right)} \int \mathrm{d} y \phi_{t_{i}, t_{i+1}}\left(\frac{C\left(t_{i}\right)}{G}, y\right) V\left(t_{i+1}, G y\right), P\left(C\left(t_{i}\right)\right)\right)
$$

Proof. The proof goes along the lines of the last section for a fraction $1 / G$ of the portfolio. The explicit expressions for the risk-free asset and the floor are simply relaxed and the lognormal cumulative law is replaced by a more general cumulative law. The Markovian property stems from the fact that the transition probability depends only on $C\left(t_{i}\right) / G$.

Remark 1. The condition on the cumulative between rebalancing dates excludes stochastic volatility and more generally models with hidden variables. In fact such models can be used within our framework at the cost of increasing the dimensionality of the Markov process by the number of additional variables. For example a stochastic volatility model for the underlying will be converted into a 2-dimensional discrete-time Markov process. 


\subsection{Numerical implementation}

The numerical implementation is rather direct from proposition 1 .

- The normalized value $C\left(t_{i}\right) / G$ of the portfolio was previously allowed to be any real number. This real line is discretized into a grid $h_{j}$, with mid-points $h_{j}^{ \pm}$such that $h_{j}<h_{j}^{+}=h_{j+1}^{-}<h_{j+1}$. The (rescaled) initial value of the portfolio $C\left(t_{0}\right) / G$ is supposed to be on the grid at node $j_{0}$ : $C\left(t_{0}\right)=G h_{j_{0}}$.

- The transition operator of equation (7) acting on real functions is replaced with a transition matrix $M_{j k}^{(i, i+1)}$ acting on vectors.

If $w\left(t_{i}, h_{j}\right)>0$ the differentiation $\partial / \partial y$ in equation (7) becomes a finite difference

$$
\begin{aligned}
M_{j k}^{(i, i+1)}= & \mathcal{Q}_{t_{i}, t_{i+1}}\left[\frac{h_{k}^{+}-\left(1-w\left(t_{i}, h_{j}\right)\right) h_{j} B\left(t_{i+1}\right) / B\left(t_{i}\right)}{w\left(t_{i}, h_{j}\right) h_{j}}\right] \\
& -\mathcal{Q}_{t_{i}, t_{i+1}}\left[\frac{h_{k}^{-}-\left(1-w\left(t_{i}, h_{j}\right)\right) h_{j} B\left(t_{i+1}\right) / B\left(t_{i}\right)}{w\left(t_{i}, h_{j}\right) h_{j}}\right]
\end{aligned}
$$

If $w\left(t_{i}, h_{j}\right)=0$ the delta function becomes the indicator function

$$
M_{j k}^{(i, i+1)}=\mathbb{1}_{\left[h_{k}^{-}, h_{k}^{+}[\right.}\left(h_{j} \frac{B\left(t_{i+1}\right)}{B\left(t_{i}\right)}\right)
$$

- For a European product, we define a vector $V_{j}^{(i)}$ with the value of the derivative at date $t_{i}$ conditionally to $C\left(t_{i}\right)=G h_{j}$. The backward propagation (8) is turned into the matrix-vector product

$$
V_{j}^{(i)}=e^{-r\left(t_{i+1}-t_{i}\right)} \sum_{k} M_{j k}^{(i, i+1)} V_{k}^{(i+1)}
$$

with terminal condition

$$
V_{j}^{(n)}=P\left(G h_{j}\right)
$$

The fair price of the derivative at time 0 is given by

$$
V\left(t_{0}, C\left(t_{0}\right)\right)=V_{j_{0}}^{(0)}
$$

- In the case of a Bermudan product the recursion is

$$
V_{j}^{(i)}=\max \left(e^{-r\left(t_{i+1}-t_{i}\right)} \sum_{k} M_{j k}^{(i, i+1)} V_{k}^{(i+1)}, P\left(G h_{j}\right)\right)
$$

\subsection{Numerical convergence}

In order to speed up the pricing, the time-dependent parameters (mainly interest rates and volatilities) are approximated by piecewise constant functions. The approximation is controlled by a tolerance number which bounds the relative error between the approximation and the real functions. This procedure greatly reduces the number of matrices to be generated.

Relative errors with respect to the most accurate case (no approximation, 2500 points) and computation times as functions of the tolerance level and of the grid size are presented in table 1 in the case of a put on a CPPI portfolio with strike at the guaranteed amount. Prices smoothly converge 
when the grid size is increased and the approximation level reduced. A precise price can be obtained 1 in about $1 \mathrm{~s}$.

\begin{tabular}{|c|ccccc|}
\hline & $0 \%$ & $5 \%$ & $20 \%$ & $50 \%$ & $100 \%$ \\
\hline 50 & $1.35 \times 10^{+1}$ & $1.35 \times 10^{+1}$ & $1.36 \times 10^{+1}$ & $1.37 \times 10^{+1}$ & $1.40 \times 10^{+1}$ \\
& 0.76 & 0.13 & 0.10 & 0.08 & 0.07 \\
100 & $-7.36 \times 10^{-2}$ & $-7.34 \times 10^{-2}$ & $-7.20 \times 10^{-2}$ & $-6.07 \times 10^{-2}$ & $-3.22 \times 10^{-2}$ \\
& 1.61 & 0.19 & 0.12 & 0.09 & 0.08 \\
250 & $-4.69 \times 10^{-2}$ & $-4.67 \times 10^{-2}$ & $-4.53 \times 10^{-2}$ & $-3.43 \times 10^{-2}$ & $-5.94 \times 10^{-3}$ \\
& 5.26 & 0.44 & 0.21 & 0.14 & 0.12 \\
500 & $-1.21 \times 10^{-2}$ & $-1.19 \times 10^{-2}$ & $-1.05 \times 10^{-2}$ & $7.52 \times 10^{-4}$ & $3.12 \times 10^{-2}$ \\
& 15.33 & 1.15 & 0.51 & 0.31 & 0.28 \\
750 & $-4.96 \times 10^{-3}$ & $-4.77 \times 10^{-3}$ & $-3.30 \times 10^{-3}$ & $8.45 \times 10^{-3}$ & $3.83 \times 10^{-2}$ \\
& 30.53 & 2.33 & 1.07 & 0.73 & 0.66 \\
1000 & $-2.53 \times 10^{-3}$ & $-2.34 \times 10^{-3}$ & $-8.90 \times 10^{-4}$ & $1.07 \times 10^{-2}$ & $4.00 \times 10^{-2}$ \\
& 50.62 & 4.38 & 2.32 & 1.74 & 1.64 \\
1500 & $-6.99 \times 10^{-4}$ & $-5.11 \times 10^{-4}$ & $9.28 \times 10^{-4}$ & $1.24 \times 10^{-2}$ & $4.15 \times 10^{-2}$ \\
2000 & 109.31 & 10.20 & 5.69 & 4.41 & 4.17 \\
& $-1.75 \times 10^{-4}$ & $1.47 \times 10^{-5}$ & $1.46 \times 10^{-3}$ & $1.29 \times 10^{-2}$ & $4.22 \times 10^{-2}$ \\
2500 & 173.11 & 17.70 & 10.46 & 8.46 & 8.17 \\
& - & $1.89 \times 10^{-4}$ & $1.62 \times 10^{-3}$ & $1.31 \times 10^{-2}$ & $4.24 \times 10^{-2}$ \\
& 266.14 & 28.97 & 18.06 & 14.99 & 14.46 \\
\hline
\end{tabular}

Table 1: Relative error on the price of a put on a non-vanilla CPPI (top numbers) and computing time in seconds (bottom) as a function of the approximation level and the grid size. Relative errors are computed with respect to the best estimate given by no approximation and 2500 points in the grid.

A more detailed discussion of various technical aspects of the numerical implementation can be found in [PL09].

\section{Profit lock-in}

\subsection{Pricing with profit lock-in}

CPPI strategies often include some profit lock-in: a fraction $\lambda$ of the performance is locked in periodically. $G$ therefore becomes a piecewise constant stochastic process $G(t)$. The floor is scaled accordingly as

$$
H\left(t_{i}\right)=e^{-r\left(t_{n}-t_{i}\right)} G\left(t_{i}\right)
$$

such that the terminal value of the CPPI portfolio should ideally at least be $G\left(t_{n}\right)$.

We denote by $t_{\ell_{I}}, I=1 \ldots N$ the subset of rebalancing dates at which the profit lock-in occurs (typically annually). For convenience, we set $\ell_{0}=0$ and $\ell_{N}=n$. Whenever the performance of the portfolio is positive between $t_{\ell_{I}}$ and $t_{\ell_{I+1}}$ the guarantee is raised to

$$
G\left(t_{\ell_{I+1}}\right)=G\left(t_{\ell_{I}}\right)+\lambda\left[C\left(t_{\ell_{I+1}}\right)-C\left(t_{\ell_{I}}\right)\right]^{+}
$$

This expression can be recast into the more general form

$$
\frac{G\left(t_{\ell_{I+1}}\right)}{G\left(t_{\ell_{I}}\right)}=f\left(\frac{C\left(t_{\ell_{I}}\right)}{G\left(t_{\ell_{I}}\right)}, \frac{C\left(t_{\ell_{I+1}}\right)}{G\left(t_{\ell_{I}}\right)}\right)
$$

with $f(x, y)$ a positive function.

\footnotetext{
${ }^{1}$ Computations were performed on a standard PC equipped with an Intel Core 26700 at 2.66 GHz and $3.25 \mathrm{~GB}$ of memory. We used the optimized Intel's Math Kernel Library to perform matrix-matrix and matrix-vector multiplications.
} 
The rebalancing rule

$$
C^{\mathrm{risky}}\left(t_{i}\right)=\max \left(m \frac{C\left(t_{i}\right)-e^{-r\left(t_{n}-t_{i}\right)} G\left(t_{i}\right)}{C\left(t_{i}\right)}, 0\right) C\left(t_{i}\right)
$$

is of the more general form

$$
C^{\text {risky }}\left(t_{i}\right)=w\left(t_{i}, \frac{C\left(t_{i}\right)}{G\left(t_{i}\right)}\right) C\left(t_{i}\right)
$$

We are concerned with the pricing of a European derivative product $V$ written on the CPPI portfolio whose terminal payoff $P$ might depend on $C$ and $G$ at maturity. The fair price at time $t_{\ell_{i}}$ can be written as the risk-neutral expectation

$$
V\left(t_{\ell_{I}}, C\left(t_{\ell_{I}}\right), G\left(t_{\ell_{I}}\right)\right)=e^{-r\left(t_{\ell_{I+1}}-t_{\ell_{I}}\right)} \mathbb{E}\left[V\left(t_{\ell_{I+1}}, C\left(t_{\ell_{I+1}}\right), G\left(t_{\ell_{I+1}}\right)\right) \mid \mathcal{F}_{t_{\ell_{I}}}\right]
$$

This problem is two-dimensional as one must know the joint law of $C$ and $G$ to apply this formula. However, beautiful simplifications occur in the case of an homogeneous payoff of the form

$$
P(C, G)=G \widetilde{P}\left(\frac{C}{G}\right)
$$

This includes the CPPI portfolio itself or options with strike in percentage of the final guarantee. Owing to the special form of the payoff we can state the following proposition.

Proposition 2. Consider the same hypothesis as in proposition 1, except for the fact that the guarantee is not constant but is rescaled at dates $t_{\ell_{I}}$ along equation (11). For a European derivative product whose payoff at maturity is given by equation (13), the fair price at date $t_{\ell_{I}}$ is homogeneous:

$$
V\left(t_{\ell_{I}}, C\left(t_{\ell_{I}}\right), G\left(t_{\ell_{I}}\right)\right)=G\left(t_{\ell_{I}}\right) \tilde{V}\left(t_{\ell_{I}}, \frac{C\left(t_{\ell_{I}}\right)}{G\left(t_{\ell_{I}}\right)}\right)
$$

and $\widetilde{V}$ satisfies the recursion formula

$$
\widetilde{V}\left(t_{\ell_{I}}, \frac{C\left(t_{\ell_{I}}\right)}{G\left(t_{\ell_{I}}\right)}\right)=e^{-r\left(t_{\ell_{I+1}}-t_{\ell_{I}}\right)} \int \mathrm{d} y \psi_{t_{\ell_{I}}, t_{\ell_{I+1}}}\left(\frac{C\left(t_{\ell_{I}}\right)}{G\left(t_{\ell_{I}}\right)}, y\right) \widetilde{V}\left(t_{\ell_{I+1}}, y\right)
$$

with the kernel

$$
\psi_{t_{\ell_{I}}, t_{\ell_{I+1}}}(x, y)=\int \mathrm{d} z \delta\left(y-\frac{z}{f(x, z)}\right) f(x, z) \phi_{t_{\ell_{I}}, t_{\ell_{I+1}}}(x, z)
$$

In this equation, $\phi_{t_{\ell_{I}}, t_{\ell_{I+1}}}(x, z)$ is the transition operator defined in proposition 1 .

Proof. At time $t_{n}$, equation (14) is verified due to equation (13). We assume now equation (14) holds at time $t_{\ell_{I+1}}$. We denote by $t_{\ell_{I+1}}^{-}$a time just before the profit lock-in, i.e. before the jump in $G(t)$, but after possible jumps in the risky asset $S(t)$ (this describes the state of the system before applying the lock-in). As there is no profit lock-in between $t_{\ell_{I}}$ and $t_{\ell_{I+1}^{-}}$, we can use proposition 1 with transition operator $\phi_{t_{\ell_{I}}, t_{\ell_{I+1}^{-}}}$and write

$$
V\left(t_{\ell_{I}}, C\left(t_{\ell_{I}}\right), G\left(t_{\ell_{I}}\right)\right)=e^{-r\left(t_{\ell_{I+1}}-t_{\ell_{I}}\right)} \int \mathrm{d} y \phi_{t_{\ell_{I}}, t_{\ell_{I+1}^{-}}}\left(\frac{C\left(t_{\ell_{I}}\right)}{G\left(t_{\ell_{I}}\right)}, y\right) V\left(t_{\ell_{I+1}^{-}}, G\left(t_{\ell_{I}}\right) y, G\left(t_{\ell_{I}}\right)\right)
$$

When the lock-in is performed, the portfolio value is not changed:

$$
V\left(t_{\ell_{I+1}^{-}}, G\left(t_{\ell_{I}}\right) y, G\left(t_{\ell_{I}}\right)\right)=V\left(t_{\ell_{I+1}}, G\left(t_{\ell_{I}}\right) y, G\left(t_{\ell_{I+1}}\right)\right)
$$


This value can be rewritten further using the recursion hypothesis (14) as

$$
V\left(t_{\ell_{I+1}^{-}}, G\left(t_{\ell_{I}}\right) y, G\left(t_{\ell_{I}}\right)\right)=G\left(t_{\ell_{I+1}}\right) \widetilde{V}\left(t_{\ell_{I+1}}, \frac{G\left(t_{\ell_{I}}\right) y}{G\left(t_{\ell_{I+1}}\right)}\right)
$$

The profit lock-in is deterministic as a function of $G\left(t_{\ell_{I}}\right), C\left(t_{\ell_{I}}\right)$ and $C\left(t_{\ell_{I+1}}\right)$. It is given by equation (11) which reads here

$$
G\left(t_{\ell_{I+1}}\right)=G\left(t_{\ell_{I}}\right) f\left(\frac{C\left(t_{\ell_{I}}\right)}{G\left(t_{\ell_{I}}\right)}, y\right)
$$

Using equations (18) and (19), equation $(17)$ is rewritten as

$$
\begin{aligned}
& V\left(t_{\ell_{I}}, C\left(t_{\ell_{I}}\right), G\left(t_{\ell_{I}}\right)\right)= \\
& \quad e^{-r\left(t_{\ell_{I+1}}-t_{\ell_{I}}\right)} G\left(t_{\ell_{I}}\right) \int \mathrm{d} y \phi_{t_{\ell_{I}}, t_{\ell_{I+1}}}\left(\frac{C\left(t_{\ell_{I}}\right)}{G\left(t_{\ell_{I}}\right)}, y\right) f\left(\frac{C\left(t_{\ell_{I}}\right)}{G\left(t_{\ell_{I}}\right)}, y\right) \widetilde{V}\left(t_{\ell_{I+1}}, \frac{y}{f\left(\frac{C\left(t_{\ell_{I}}\right)}{G\left(t_{\ell_{I}}\right)}, y\right)}\right)
\end{aligned}
$$

Inserting

$$
\int \mathrm{d} z \delta\left(z-\frac{y}{f\left(\frac{C\left(t_{\ell_{I}}\right)}{G\left(t_{\ell_{I}}\right)}, y\right)}\right)=1
$$

we find that $V\left(t_{\ell_{I}}, C\left(t_{\ell_{I}}\right), G\left(t_{\ell_{I}}\right)\right)$ is indeed of the form given by equation (14) with equations (15) and (16) satisfied.

Remark 2. Proposition 2 can be generalized to a payoff homogeneous of degree $\alpha$ :

$$
P(C, G)=G^{\alpha} \widetilde{P}\left(\frac{C}{G}\right)
$$

The only modifications are in equation 116 where $G\left(t_{\ell_{I}}\right)$ is replaced with $G\left(t_{\ell_{I}}\right)^{\alpha}$ and in equation $(16)$ where $f(x, z)$ is replaced with $f(x, z)^{\alpha}$.

Remark 3. Proposition 2 can also be generalized to a Bermudan payoff with exercise allowed at lock-in or reallocation dates. The maximum of values with and without exercising has simply to be introduced in the backward propagation.

\subsection{Numerical implementation}

The numerical implementation of proposition 2 is straightforward, building on the basis of section 2.3 .

- A grid is built as in the basic case of section 2.3, such that $C\left(t_{0}\right) / G\left(t_{0}\right)=h_{j_{0}}$ for some index $j_{0}$.

- From the local transition matrices $M_{j k}^{(i, i+1)}$ of section 1 , we compute the transition matrices between two lock-in dates as the matrix product

$$
M^{\left(\ell_{I}, \ell_{I+1}\right)}=M^{\left(\ell_{I}, \ell_{I}+1\right)} M^{\left(\ell_{I}+1, \ell_{I}+2\right)} \ldots M^{\left(\ell_{I+1}-1, \ell_{I+1}\right)}
$$


- Using the lock-in function $f(x, y)$, the discrete analogue of the kernel $\psi_{t_{\ell_{I}}, t_{\ell_{I+1}}}(x, y)$ of equation (16) is the matrix

$$
N_{j k}^{(I, I+1)}=\sum_{l} \mathbb{1}_{\left[h_{k}^{-}, h_{k}^{+}[\right.}\left(\frac{h_{l}}{f\left(h_{j}, h_{l}\right)}\right) f\left(h_{j}, h_{l}\right) M_{j l}^{\left(\ell_{I}, \ell_{I+1}\right)}
$$

- The rescaled payoff $\widetilde{V}$ of proposition 2 is discretized as $\widetilde{V}_{j}^{(I)}=\tilde{V}\left(t_{\ell_{I}}, h_{j}\right)$. It is propagated backward from lock-in date to lock-in date through equation (15) which becomes

$$
\widetilde{V}_{j}^{(I)}=e^{-r\left(t_{\ell_{I+1}}-t_{\ell_{I}}\right)} \sum_{k} N_{j k}^{(I, I+1)} \widetilde{V}_{k}^{(I+1)}
$$

with terminal condition

$$
\widetilde{V}_{j}^{(N)}=\widetilde{P}\left(h_{j}\right)
$$

- The fair price of the derivative at time 0 is given by

$$
V\left(t_{0}, C\left(t_{0}\right), G\left(t_{0}\right)\right)=G\left(t_{0}\right) \widetilde{V}_{j_{0}}^{(0)}
$$

\subsection{Probability transition}

Proposition 2 provides a pricing algorithm for payoff which are homogeneous in $C, G$. The distribution of the portfolio value can also be computed, which may be useful for valuation and risk management of options with strike given as a fixed amount or for computing the final probability density function of a CPPI portfolio.

Proposition 3. The probability transition operator between two lock-in dates $t_{\ell_{I}}$ and $t_{\ell_{J}}$

$$
\Phi_{t_{\ell_{I}}, t_{\ell_{J}}}(x, y)=\mathbb{E}\left[\delta\left(\frac{C\left(t_{\ell_{J}}\right)}{G\left(t_{\ell_{I}}\right)}-y\right) \mid \frac{C\left(t_{\ell_{I}}\right)}{G\left(t_{\ell_{I}}\right)}=x\right]
$$

can be computed from the transition operator without profit lock-in as

$$
\Phi_{t_{\ell_{I}}, t_{\ell_{J}}}(x, y)=\int \mathrm{d} z \frac{1}{f(x, z)} \phi_{t_{\ell_{I}}, t_{\ell_{I+1}}}(x, z) \Phi_{t_{\ell_{I+1}}, t_{\ell_{J}}}\left(\frac{z}{f(x, z)}, \frac{y}{f(x, z)}\right)
$$

Proof. This result is a rewriting of

$$
\begin{aligned}
& \mathbb{E}\left[\delta\left(\frac{C\left(t_{\ell_{J}}\right)}{G\left(t_{\ell_{I}}\right)}-y\right) \mid \frac{C\left(t_{\ell_{I}}\right)}{G\left(t_{\ell_{I}}\right)}=x\right]= \\
& \quad \int \mathrm{d} z \mathbb{E}\left[\delta\left(\frac{C\left(t_{\ell_{I+1}}\right)}{G\left(t_{\ell_{I}}\right)}-z\right) \mid \frac{C\left(t_{\ell_{I}}\right)}{G\left(t_{\ell_{I}}\right)}=x\right] \mathbb{E}\left[\delta\left(\frac{C\left(t_{\ell_{J}}\right)}{G\left(t_{\ell_{I+1}}\right)} \frac{G\left(t_{\ell_{I+1}}\right)}{G\left(t_{\ell_{I}}\right)}-y\right) \mid \frac{C\left(t_{\ell_{I+1}}\right)}{G\left(t_{\ell_{I+1}}\right)} \frac{G\left(t_{\ell_{I+1}}\right)}{G\left(t_{\ell_{I}}\right)}=z\right]
\end{aligned}
$$

using equation (11).

Supposing for simplicity that $\ell_{0}=0$ and $\ell_{N}=n$, the distribution of the portfolio at final maturity $t_{n}$ can then be obtained as

$$
\mathbb{E}\left[\delta\left(C\left(t_{n}\right)-y\right) \mid C\left(t_{0}\right)=x\right]=\frac{1}{G\left(t_{0}\right)} \Phi_{t_{0}, t_{n}}\left(\frac{x}{G\left(t_{0}\right)}, \frac{y}{G\left(t_{0}\right)}\right)
$$

The main difference with the homogenous payoff is the following: whereas the dynamics of $C\left(t_{\ell_{I}}\right) / G\left(t_{\ell_{I}}\right)$ taken at lock-in dates is Markovian, it is not true for the dynamics of $C\left(t_{\ell_{I}}\right)$ itself.

The numerical implementation is similar to the other cases. Equation (20) is not a product of operators. As a consequence, it is also numerically more time-consuming. 


\section{$4 \quad$ Numerical examples}

In order to illustrate the smoothness of the results, we plot in figure 2 the probability distribution at maturity for three different CPPI strategies. We take a risky asset which follows the jump-diffusion model introduced by Kou Kou02. There are upward and downward jumps, both exponentially distributed with distinct parameters. The diffusion has volatility $20 \%$, downward jump with intensity $0.1 \mathrm{yr}^{-1}$ and mean value 0.1 , upward jumps with intensity $0.1 \mathrm{yr}^{-1}$ and mean value 0.05 . We consider strategies on this risky asset with a maturity of 10 years and weekly rebalancing. The risk-free rate gives an initial zero-coupon value of 0.606 .

- We consider first the vanilla case of section 2 with multiplier $m=4$ :

$$
w(t, C)=\max \left(m \frac{C-H(t)}{C}, 0\right)
$$

- We add a cap (150\%) to the risky asset exposure so that

$$
w(t, C)=\min \left(\operatorname{Cap}, \max \left(m \frac{C-H(t)}{C}, 0\right)\right)
$$

- Removing the cap, we finally incorporate to the strategy an annual lock-in of $75 \%$ as described in section 3 .

We refer the reader to PL09] for further discussions of the effects of the various features commonly found in CPPI-based contracts.

\section{Conclusion}

We introduced an efficient scheme for the pricing of CPPI strategies and options. Instead of following the underlying spot, the portfolio value, the risky asset weighting and the guaranteed amount as in a Monte-Carlo pricing or a classical PIDE backward propagation, only the CPPI portfolio value at rebalancing dates is considered (or the ratio of the CPPI over the guaranteed amount in the case of lock-in). We proved that under the hypothesis of homogenous behavior of the underlying, the portfolio value at rebalancing dates (or lock-in dates) is a discrete time Markov process in one single variable. We derived a natural pricing scheme which uses this property to price both European and Bermudan derivatives on the CPPI portfolio. This allows in particular to estimate the gap risk of guaranteed CPPI products or even to price options on CPPI strategies.

\section{Acknowledgements}

We thank Erwan Curien, Martial Millet and Quentin Serres for useful remarks on this work. 

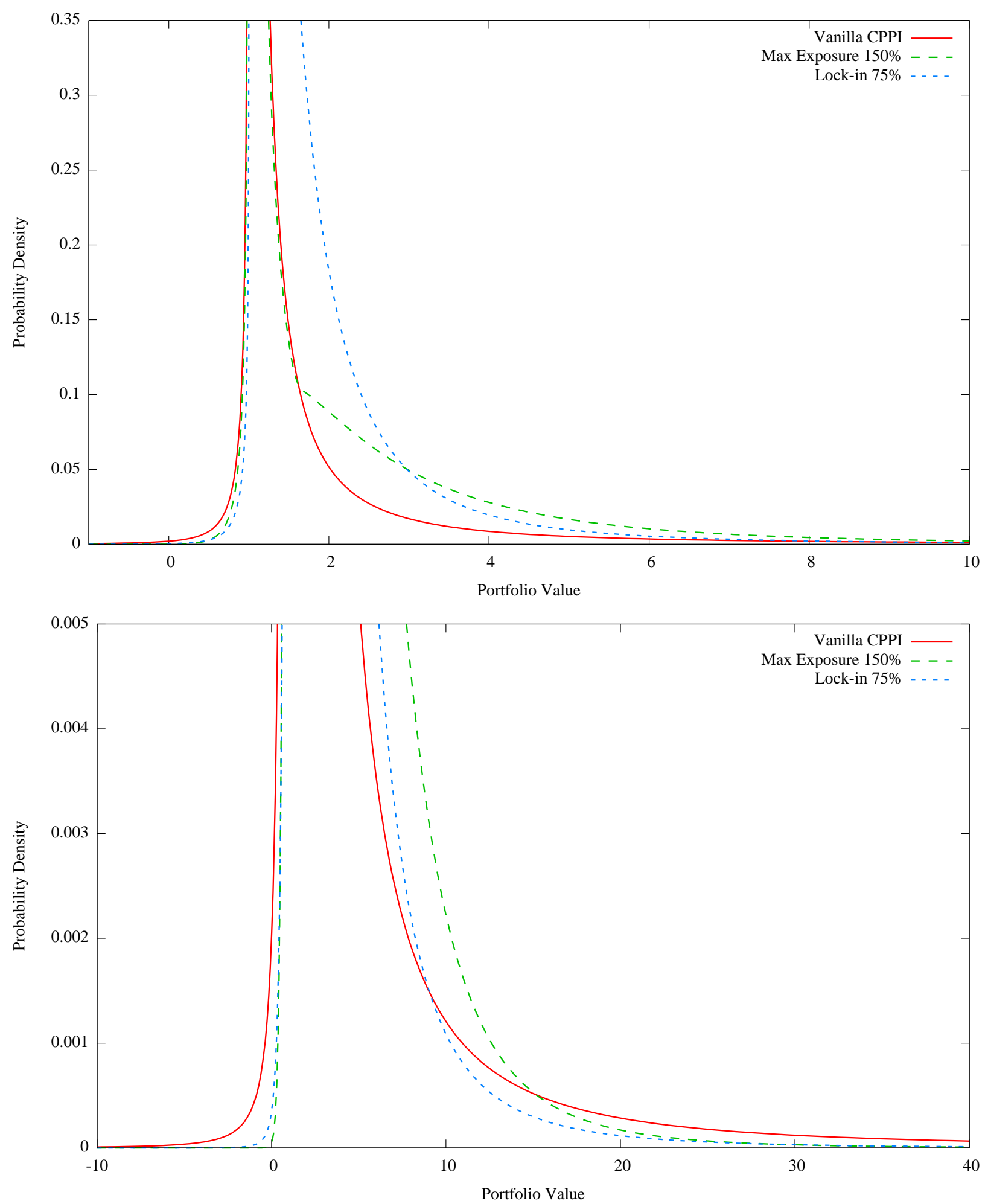

Figure 2: Probability Density of a CPPI portfolio value at maturity for three different strategies. For the first curve, the CPPI has maturity 10 years, weekly rebalancing, multiplier 4. The initial investment and the initial guaranteed level are 1. The mean price of the strategy is 1.65. The risky asset follows a Brownian motion with 20\% constant volatility with additional jumps (Kou model): downward jump with intensity $0.1 \mathrm{yr}^{-1}$ and mean value 0.1, upward jumps with intensity $0.1 \mathrm{yr}^{-1}$ and mean value 0.05. For the second curve the risky asset weighting is capped to 150\%. For the third one there is no cap on the exposure but a profit lock-in of $75 \%$ of the performance is performed annually. The second graph is a zoom on the tails of the distribution. 


\section{References}

[BBM05] Sven Balder, Michael Brandl, and Antje Mahayni. Effectiveness of CPPI strategies under discrete time trading. http://ssrn.com/abstract=898625, 2005.

[BK95] Jean-François Boulier and Anu Kanniganti. Expected performance and risk of various portfolio insurance strategies. In Proceedings of the 5th AFIR International Colloquium, pages 1093-1124, Brussels, 1995. http://www.actuaries.org/AFIR/colloquia/ Brussels/Boulier_Kanniganti.pdf.

[BP02] Philippe Bertrand and Jean-Luc Prigent. Portfolio insurance: the extreme value approach to the CPPI method. Finance, 23(2):69-86, 2002. http://ssrn. com/abstract=299690.

[CT07] Rama Cont and Peter Tankov. Constant proportion portfolio insurance in presence of jumps in asset prices. http://ssrn.com/abstract=1021084, 2007.

[Kou02] Steven G. Kou. A jump-diffusion model for option pricing. Management Science, 48(8):1086-1101, 2002. http://ssrn.com/abstract=242367

[Mer71] Robert C. Merton. Optimum consumption and portfolio rules in a continuous-time model. Journal of Economic Theory, 3(4):373-413, 1971.

[PL09] Louis Paulot and Xavier Lacroze. Efficient pricing of CPPI using Markov operators. http: //arxiv.org/abs/0901.1218, 2009. 